\title{
An Open Source Quality Assurance Tool for HL7 v2 Syndromic Surveillance Messages
}

\author{
Noam H. Arzt* and Srinath Remala
}

HLN Consulting, LLC, Palm Desert, CA, USA

\section{Objective}

To leverage an existing open source quality assurance software tool created for the immunization domain and modify it to serve as a quality assurance tool for syndromic surveillance messages.

\section{Introduction}

The CMS EHR Incentive Programs include a measure for meaningful use of EHR systems for submitting syndromic surveillance messages to public health [1]. The Stage 2 measure defines the standard for transmission to be HL7 v2.5.1 Admit/Discharge/Transfer messages according to the PHIN Messaging Guide for Syndromic Surveillance and Conformance Clarification for EHR Certification of Electronic Syndromic Surveillance, Addendum to PHIN Messaging Guide for Syndrome Surveillance [2]. The National Institute of Standards and Technology (NIST) provides an online testing tool for validating messages [3]. While some jurisdictions use the Biosense platform for receiving, managing, and analyzing syndromic surveillance data, there is no consistent tool that is available to jurisdictions to assess the quality and conformance of data submissions both at the time of on-boarding a new reporting facility and on an ongoing basis during production operations [4].

The New York City Citywide Immunization Registry (CIR), the immunization information system for NYC that has been operational since 1997, has as part of its software suite an Open Source, webbased data quality assurance (QA) tool used by its research scientists to qualify new sites for reporting data electronically via HL7 v2 messages, and for monitoring the ongoing quality of data submissions over time [5]. A validation process evaluates incoming messages against the rules established by an implementation guide (IG) and stores the result of the evaluation in a CIR database table that is accessible by the QA Tool which displays the data to an administrative user. This project served as a proof-of-concept for implementing a similar process for syndromic surveillance.

\section{Methods}

Building upon existing Open Source software, the project developed a self-contained, end-to-end prototype of the quality assurance system for syndromic surveillance. The resulting product has two main components:

1. A data validation process, using a local instance of NIST HL7 v2 Validation Tool and repackaged to accept a sample HL7 v2.5.1 ADT message via file upload, evaluate it against the PHIN Guide, and store both the raw message and any errors or warnings generated in a local CIR compliant database.

2. An enhanced version of the CIR QA Tool that reads the raw messages, errors and warnings from the previous step and allows users to query and display messages for a specific reporting facility and date range.

\section{Results}

The system was created and deployed using open source software. Various test messages, based on NIST test data set, were sent through the system and the results were examined against known outcomes. The user interface required only minor modification (Figure) to accommodate the change in message type from VXU to ADT.

\section{Conclusions}

This project demonstrated the successful leverage of public health developed, Open Source software from one domain to another. It also established new capacity for public health agencies to monitor the quality of data submissions for syndromic surveillance. Future enhancements to this product might include:

- Tighter integration with BioSense through export of "passing" messages into formats that can be imported into the BioSense platform.

- Enhancement of the summary statistics and reports available for message batches.

- More complete role-based security to recognize differing staff responsibility for different parts of the public health agency workflow that this product supports.

- User-configurable support for variations in the implementation guide specifications used to evaluate message and present errors and warnings.

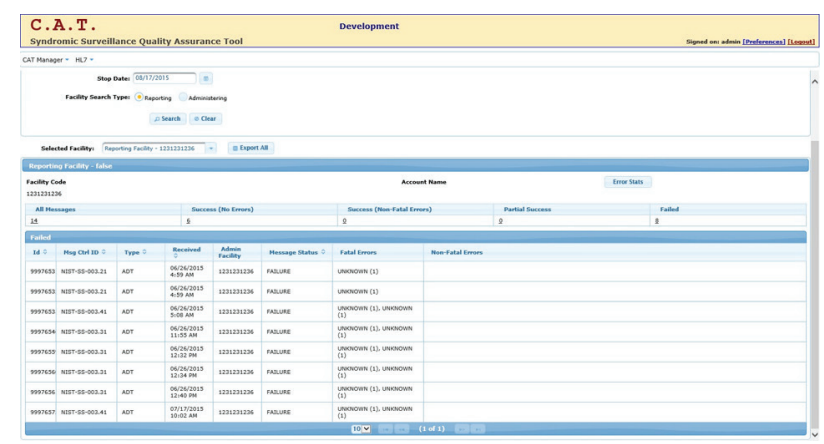

\section{Keywords}

HL7; ADT; syndromic surveillance

\section{Acknowledgments}

The authors are thankful both the Centers for Disease Control and Prevention, Center for Surveillance, Epidemiology, and Laboratory Services (CDC/CSELS) for funding this work, and the Association of State and Territorial Health Officers (ASTHO) under whose auspices this project was conducted.

\section{References}

[1] http://www.cms.gov/Regulations-and-Guidance/Legislation/ EHRIncentivePrograms/

[2] http://www.cdc.gov/phin/resources/PHINguides.html\#ss

[3] http://h17v2-ss-testing.nist.gov/mu-syndromic/

[4] http://www.cdc.gov/biosense/

[5] http://www.nyc.gov/html/doh/html/hcp/cir.shtml

\section{*Noam H. Arzt}

E-mail: arzt@hln.com 